\title{
Le bassin rhénan et le Nord : histoire du fonds scandinave de la Bibliothèque nationale et universitaire de Strasbourg
}

\section{Pierre-Brice Stahl}

\section{(2) OpenEdition}

Journals

Édition électronique

URL : https://journals.openedition.org/rbnu/1971

DOI : 10.4000/rbnu. 1971

ISSN : 2679-6104

Éditeur

Bibliothèque nationale et universitaire de Strasbourg

\section{Édition imprimée}

Date de publication : 1 novembre 2013

Pagination : 38-43

ISBN : 9782859230517

ISSN : 2109-2761

\section{Référence électronique}

Pierre-Brice Stahl, « Le bassin rhénan et le Nord : histoire du fonds scandinave de la Bibliothèque nationale et universitaire de Strasbourg », La Revue de la BNU [En ligne], 8 | 2013, mis en ligne le 01 novembre 2013, consulté le 19 mai 2021. URL : http://journals.openedition.org/rbnu/1971 ; DOI : https://doi.org/10.4000/rbnu.1971 


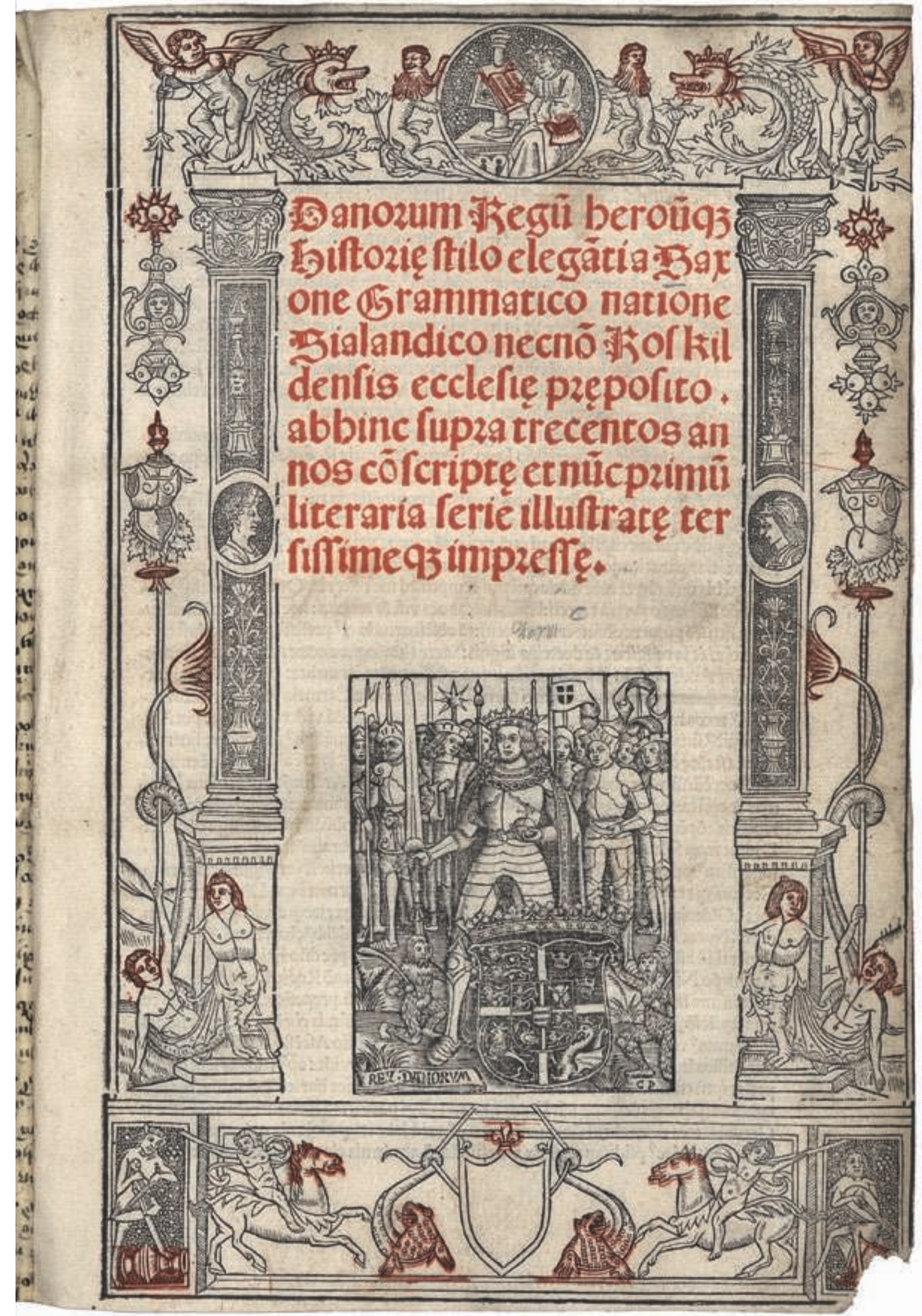

Page de titre de la Gesta Danorum de Saxo Grammaticus (Paris, Josse Bade, 1514; coll. BNU) 


\section{Le bassin rhénan et le Nord :}

\section{histoire du fonds scandinave de la Bibliothèque nationale et universitaire de Strasbourg}

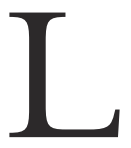

e fonds scandinave ancien de la BNU comprend plus d'un millier d'ouvrages antérieurs au $19^{\mathrm{e}}$ siècle. Soit ces derniers traitent du Nord, soit ils sont originaires d'un des pays scandinaves (Norvège, Danemark ou Suède). Ainsi, environ $23 \%$ des ouvrages proviennent du Danemark (plus précisément de Copenhague) et $17 \%$ de la Suède (Stockholm et Upsal étant les principaux centres de publication) ${ }^{1}$. Les sujets traités vont de la botanique à la mythologie en passant par les guerres du Nord ; l'on y retrouve de grands noms tels que ceux de Johannes Schefferus, Olaus Magnus, Christine de Suède entre autres. Au-delà de leur contenu, ces œuvres sont également le témoin des échanges et des liens, qu'ils soient d'ordre public ou privé, entre le bassin rhénan et le Nord. Parcourir ce fonds scandinave revient à entreprendre un voyage, voyage dans le temps, voyage dans l'espace - voyage dans lequel je me propose de servir de guide, ou du moins de montrer les directions possibles à emprunter.

Toutefois, l'histoire de ce fonds a une origine tragique : celle d'un incendie. En 1870, la bibliothèque de la Ville de Strasbourg et celle du séminaire protestant furent victimes de la guerre franco-allemande et détruites dans la nuit du 24 au 25 août $^{2}$. Les collections, entreposées au Temple Neuf, contenaient alors un fonds d'ouvrages très riche, estimé à 300000 volumes. On y trouvait notamment l'Hortus deliciarum, le célèbre manuscrit alsacien du $12^{\mathrm{e}}$ siècle. C'est cette même année que suite à la guerre, l'Alsace et la Lorraine furent annexées à l'Empire allemand. Un projet se mit alors en place : la création de la Kaiserliche Universitäts- und Landesbibliothek (Bibliothèque impériale de l'université et de la région) en remplacement de l'institution perdue, et parallèlement à la mise en place de la nouvelle université de Strasbourg. Un de ses principaux instigateurs fut le bibliothécaire Karl August Barack. Il organisa notamment un appel aux dons afin de reconstituer une riche collection pour la nouvelle bibliothèque. Ce fut un succès aussi bien en Allemagne qu'à l'étranger, et des livres qui occupaient autrefois les rayons de bibliothèques allemandes, italiennes, françaises, américaines vinrent reconstituer le fonds de Strasbourg. Le fonds scandinave ancien actuellement disponible à la BNU provient en grande partie de cet appel. Il est ainsi possible de tenir entre ses mains des ouvrages ayant appartenu à la collection privée de Guillaume ${ }^{\text {er }}$, qui fit don d'environ 4000 ouvrages, ou qui auparavant garnissaient les rayons de bibliothèques d'Oxford. Ces ouvrages, qui vont de la riche reliure à la simple couverture en carton, recèlent une grande richesse par leur contenu ou leur provenance. Même si les langues principales du fonds ainsi reconstitué restent le latin et l'allemand, le lecteur intéressé par d'autres aires linguistiques, comme nous allons le voir, n'est pas pour autant au bout de ses surprises.

Ainsi, l'appel de Barack fit entrer à la bibliothèque l'édition originale de l'ouvrage de Saxo Grammaticus, la Gesta Danorum ${ }^{3}$, ouvrage le plus ancien de son fonds scandinave (il fait partie de la trentaine d'ouvrages antérieurs au $17^{\mathrm{e}}$ siècle de ce fonds). La présente édition de la Gesta Danorum est le texte ancien le plus complet qui nous soit connu, le manuscrit du $12^{\mathrm{e}}$ siècle à la base de l'élaboration de l'œuvre ayant disparu. L'ouvrage, en latin, fut publié en 1514 par Christiern Pedersen, humaniste danois. Il s'agit d'une historiographie du Danemark allant des origines à l'époque de son auteur, Saxo Grammaticus. Il est difficile de reconstituer la vie de ce dernier; on sait qu'il a vécu entre la fin du $12^{\mathrm{e}}$ et le début du $13^{\mathrm{e}}$ siècle. Dans son ouvrage, il décrit des personnages connus de l'amateur de mythologie tels qu'Odin ou Baldr. Cependant, à l'instar 
du prologue de l'Edda de Snorri que nous verrons plus loin, ceux-ci sont empreints d'évhémérisme : Saxo ne les considère pas comme des dieux, mais comme des hommes.

D'autres ouvrages de la bibliothèque contiennent également des récits mythologiques : ainsi par exemple ceux des deux frères Jacob et Wilhelm Grimm, connus pour leur collection de contes et de légendes. Parmi les plus célèbres, l'on peut citer Blanche-Neige, Cendrillon, $\mathrm{La}$ Belle au bois dormant, repris et adaptés en longs métrages d'animation par Walt Disney, mais encore Hansel et Gretel ou Le Joueur de flutte de Hamelin. Moins connu du grand public est leur apport dans le domaine de la mythologie nordique. Ils sont ainsi les auteurs des Lieder der alten Edda, parus en 1815, une traduction de poèmes eddiques présents à la BNU dans cette édition ${ }^{4}$. L'Edda poétique, à ne pas confondre avec l'Edda de Snorri, est un corpus de poèmes mythologiques et héroïques (également nommés poèmes eddiques) compilés entre autres dans le Codex Regius, un manuscrit islandais du $13^{\mathrm{e}}$ siècle. Il s'agit d'une des sources de notre connaissance de la mythologie nordique. L'autre source principale est l'Edda de Snorri précédemment mentionnée.

L'œuvre fut rédigée elle aussi au $13^{\mathrm{e}}$ siècle par l'historien et politicien islandais Snorri Sturluson. Il s'agit d'un manuel depoésiequi introduit auxbases nécessaires à la composition et retrace de nombreux mythes tels que l'origine du monde ou la fabrication des principaux objets divins (qui ont pour origine une poignée de cheveux). L'apport littéraire de Snorri ne se limite pas à son Edda ; il est également l'auteur d'un recueil contenant seize sögur (pluriel de saga) relatant l'histoire des rois de Norvège : la Heimskringla. Tous ces ouvrages, qui sont à la base de notre connaissance des mythologies nordiques, se retrouvent dans le fonds scandinave de la BNU. La bibliothèque possède ainsi certaines des plus anciennes éditions de l'Edda poétique et de l'Edda de Snorri, de même que de magnifiques fac-similés de la collection Corpus codicum islandicorum medii aevi, qui permettent de contempler, entre autres, le Codex Regius ou encore une collection de manuscrits enluminés 5 .

Le fonds présente également plus de 160 livres, manifestes, rapports sur les " guerres du Nord ». On entend par là une série de conflits ayant eu lieu en Europe du Nord et du Nord-Est aux $16^{\mathrm{e}}$ et $17^{\mathrm{e}}$ siècles. La " première guerre du Nord " se déroula entre 1655 et 166o, et opposa entre autres la Suède à la double monarchie Danemark-Norvège, à la Pologne-Lituanie et à la Russie. La " grande guerre du Nord " quant à elle vit s'affronter la Russie et la Suède de 1700 à 1721, avec différents belligérants connexes dont le Danemark, qui apporta son soutien à la Russie. C'est notamment à la suite de cette guerre que la Russie étendit sa puissance en Europe. Il est possible de suivre tous ces péripéties, événements, accords, déclarations de guerre au travers de lettres, missives, manifestes et ouvrages. Le fonds possède notamment la Réponse au manifeste que le roi de Danemark a fait publier, pour justifier la guerre qu'il fait à la Suède, datée de 1710 et offrant le point de vue des adversaires de la Suède ; les Motifs qui ont engagé Sa Majesté le Roy de Prusse, à se charger du sequestre de la Pomeranie Suédoise, et qui l'obligent à en maintenir les conditions, daté de 1715 ; ou encorel'ouvrage d'Henri-Philippe Limiers de 1721, Histoire de Suède sous le règne de Charles XII. Où l'on voit aussi les révolutions arrivées en différens tems dans ce royaume; toute la guerre du Nord. Le lecteur à la recherche d'autres intrigues politiques ne sera pas déçu. En effet, celles-ci ne se limitent pas aux seules " guerres du Nord ". À l'instar des romans policiers contemporains de Stieg Larsson, Ake Edwardson ou Camilla Läckberg, le fonds scandinave ancien livre son compte d'intrigues, de secrets, d'assassinats. À la question : " qui fut le meurtrier de Gustave III ? ", le lecteur pourra trouver une réponse dans l'ouvrage d'Artaud de Montor, Histoire de l'assassinat de Gustave III, roi de Suède. Par un officier polonais, témoin oculaire, publié en 1795. Ces intrigues politiques ne sont pas uniquement liées à la guerre, comme l'illustre bien l'ouvrage de ChristianGottfried Franckenstein, Histoire des intrigues galantes de la reine Christine de Suède et de sa Cour, pendant son Séjour à Rome, publié en 1697. Franckenstein, en l'occurrence, n'en est pas l'auteur : il a utilisé différents manuscrits dont il avait fait l'acquisition auprès d'un abbé français qui était établi à Rome. L'auteur du récit est un officier de la reine Christine, qui dans son manuscrit laisse transparaître sa colère à l'égard du cardinal Decio Azzolino, qui hérita 

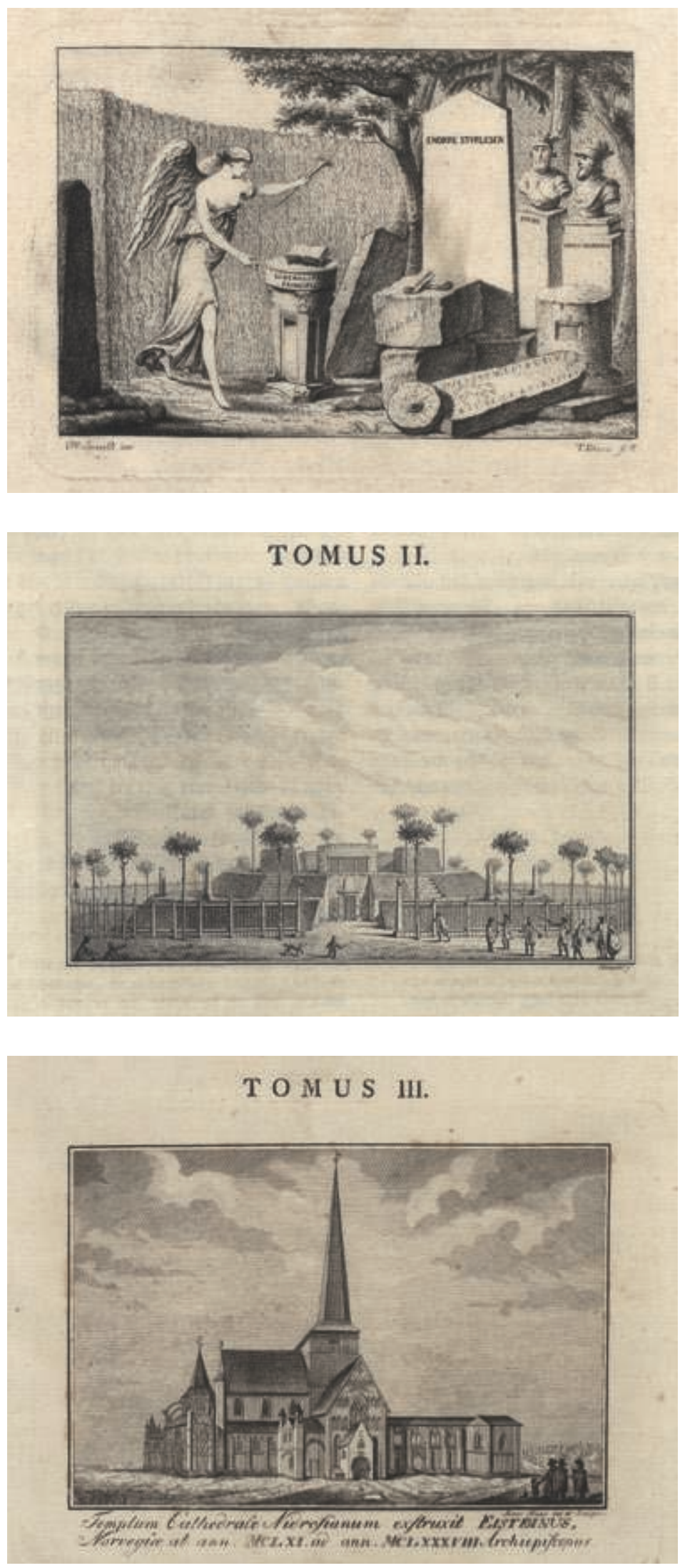

Vignettes ornant les pages de titre des trois premiers volumes de l'Heimskringla de Snorri Sturluson (édition de Copenhague, 1777-1783 ; coll. BNU) 
des biens de Christine à la mort de cette dernière. Dans sa préface au lecteur, Franckenstein note que le manuscrit est " digne de la presse " et qu'il relate des événements " dont personne n'avait eu jusqu'icy aucune connoissance " ${ }^{6}$. Le récit ne doit pas cependant être toujours pris au pied de la lettre et l'on peut s'interroger sur la véracité de certaines allégations (ainsi l'affaire entre la reine et Henri Ir $^{\text {er }}$ de Guise). Un des principaux traits qui ressort de la lecture de ce récit est l'érudition de la reine. Le fonds scandinave de la BNU en témoigne d'ailleurs au travers des différentes correspondances qu'elle entretenait, notamment avec Descartes, Pascal ou encore Louis XIV.

Dans notre imaginaire collectif, le Nord n'est cependant pas immédiatement associé à ces conflits et intrigues. Il est plus généralement synonyme de nature, d'évasion et de liberté. On retrouve ainsi, rédigés bien avant les récits de voyage de Nicolas Vanier, les titres d'Eggert Ólafsson, La Martinière, Linné ou encore Johannes Schefferus (voir à ce sujet aussi l'article de Thomas Mohnike). En tout, le fonds de la BNU dispose de plus d'une centaine d'ouvrages antérieurs à 1825 sur le sujet. Les titres de ces ouvrages sont déjà représentatifs de ces paysages présents dans notre fonds de représentation commun. Il suffit de prendre l'exemple des récits d'Eggert Ólafsson (1726-1768), publiés en français en 1802 sous le titre de Voyage en Islande, fait par ordre de S. M. Danoise, contenant des observations sur les mœurs et les usages des Habitants; une description des Lacs, Rivières, Glaciers, Sources Chaudes et Volcans; des diverses espèces de Terres, Pierres, Fossiles et Pétrifications; des Animaux, Poissons et Insectes, etc., etc. ; avec un atlas ${ }^{7}$. L'ouvrage, qui présente une description approfondie du pays et de ses habitants, est le fruit de ses recherches au cours de voyages effectués en Islande entre 1752 et 1757. Eggert Ólafsson était à la fois un écrivain, un scientifique, un poète et un patriote. Ces deux derniers aspects se traduisent au mieux dans son poème Ofsjónir (1752) où il est le premier à décrire celle qui va devenir la Marianne islandaise, à savoir Fjallkonan, la "Dame des montagnes".

Mais revenons aux récits d'Eggert Ólafsson traduits en 1802. Sur la page de titre l'on peut lire : «A Paris, Chez les Frères Levrault, Libraires, quai Malaquai [sic] ; Et à Strasbourg, chez les mêmes ". Strasbourg, la capitale alsacienne, que l'on retrouve également sous le nom d'Argentoratum dans de nombreux récits antérieurs au $18^{\mathrm{e}}$ siècle, est présentée comme lieu de production et de diffusion dans environ $4 \%$ des ouvrages du fonds de la BNU. Ces derniers sont un des témoins de la place qu'occupait l'Alsace et des liens qu'elle entretenait avec le Nord. Strasbourg était un carrefour du savoir. Cette place internationale occupée par la ville est entre autres due à son université et à ses enseignants, dont le célèbre professeur et historien Jean-Daniel Schoepflin. Même si aucun de ses voyages ne semble l'avoir amené au Danemark, en Suède ou en Norvège, il est en lien avec des savants scandinaves (il s'est d'ailleurs vu offrir une chaire à l'université d'Upsal en Suède). Il illustre cette époque où l'échange des idées se fait notamment entre académies. Les contacts personnels des enseignants de l'université de Strasbourg jouèrent un grand rôle dans son attrait international. Les associations, les sociétés et les académies savantes participent de la diffusion du savoir de l'époque : entretenir des liens est essentiel. Strasbourg en tant que ville rhénane présentait également un avantage linguistique. L'attrait pour l'Alsace avait bien sûr aussi à voir avec cette double culture, française et germanique, qui permettait l'usage des deux langues auxquelles s'adjoignait, bien sûr, le latin pour les érudits. Enfin, la ville qui regroupait aussi les deux confessions, catholique et protestante, favorisait les échanges avec d'autres universités protestantes de l'Europe, dont les universités de Suède et du Danemark. On dénombre ainsi plus de 300 étudiants d'origine nordique qui étudièrent à l'université de Strasbourg aux $17^{\mathrm{e}}$ et $18^{\mathrm{e}}$ siècles. Ils viennent principalement du Danemark et de la Suède, mais on compte également des Norvégiens et des Islandais. Tous ces éléments représentèrent un atout sur le plan intellectuel et favorisèrent les échanges qui permirent à Strasbourg d'être une plateforme européenne en lien avec le Nord.

La mythologie, les guerres du Nord, les intrigues politiques et les récits de voyage ne sont qu'une partie des sujets qu'offre à ses lecteurs le fonds scandinave de la BNU. Le projet de valorisation de ce fonds s'est concrétisé par la numérisation de 200 ouvrages qui seront dès lors préservés numériquement et disponibles sur Internet. La collaboration entre différents chercheurs et instituts européens que cette opération a occasionnée témoigne de la place qu'occupe toujours le bassin rhénan comme plateforme de lien, de communication et de contacts avec le Nord, faisant de Strasbourg, avec son fonds scandinave, non seulement un lieu du savoir, mais également un lieu $\mathrm{du}$ " faire savoir ».

\section{Pierre-Brice Stahl}




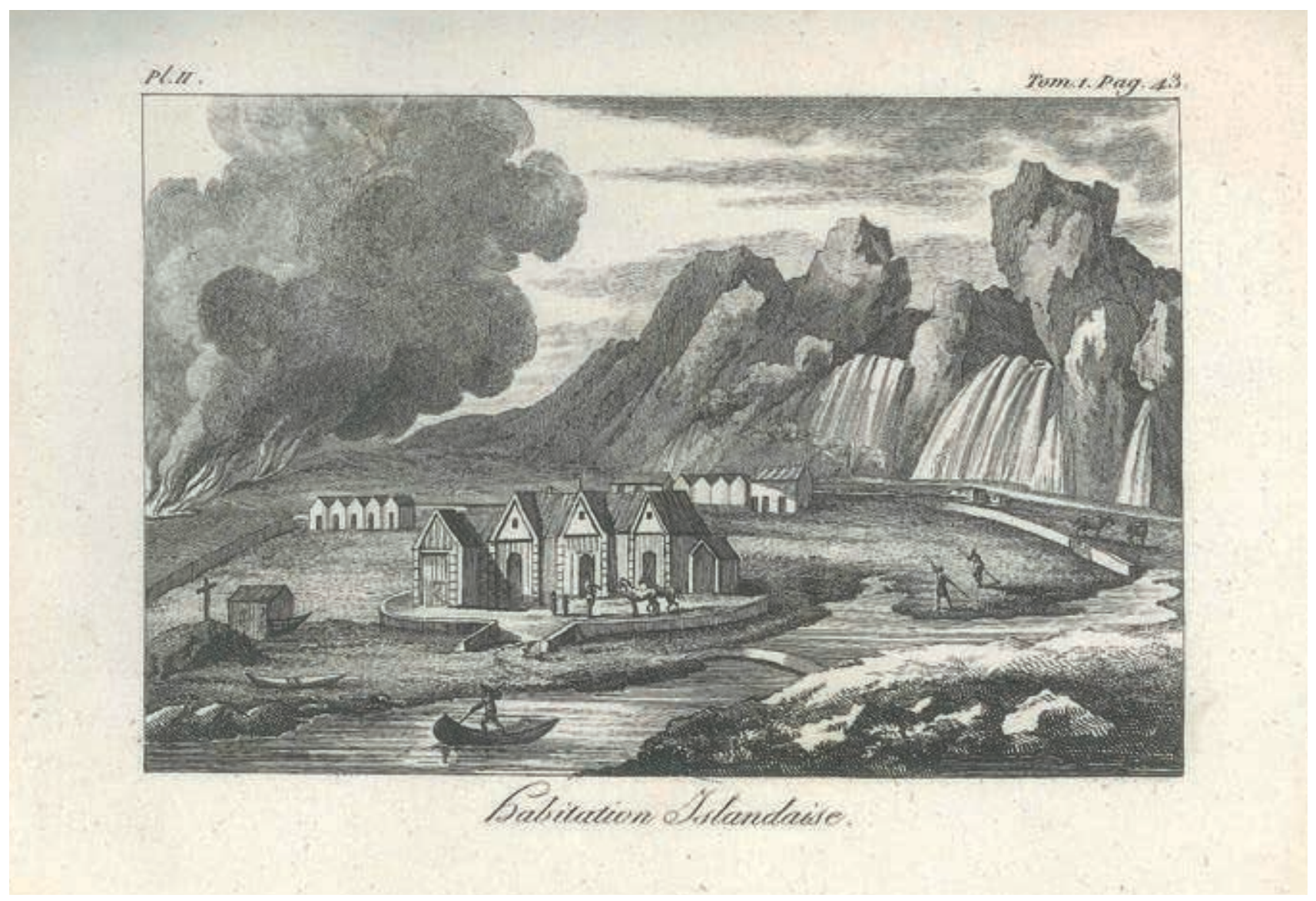

Planche représentant une habitation islandaise, extraite de l'Atlas du voyage en Islande, fait par ordre de S. M. Danoise (édition de Paris et Strasbourg, 1802 ; coll. BNU)

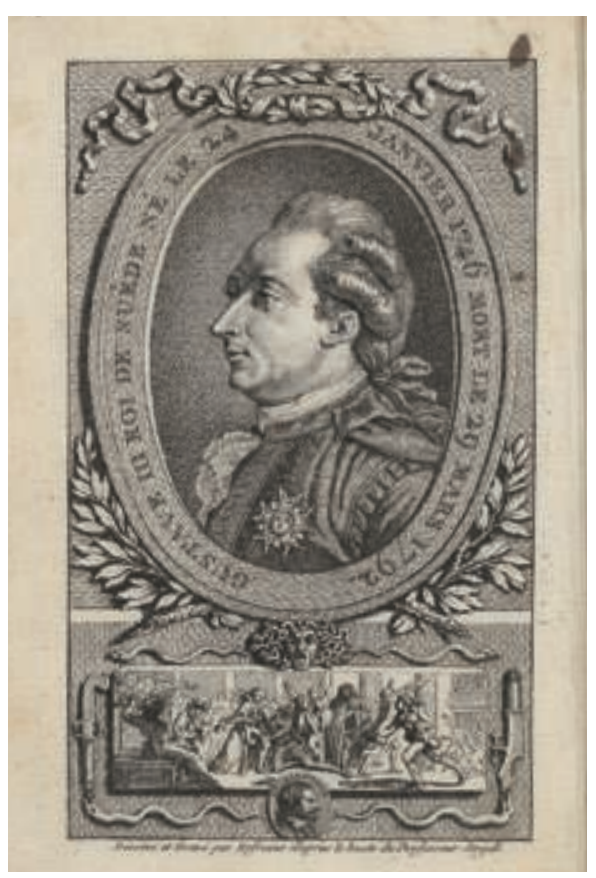

Portrait de Gustave III, placé en frontispice de l'ouvrage Histoire de l'assassinat de Gustave III, roi de Suède (édition de Paris, 1797 ; coll. BNU)

\section{Notes}

1 - Les données présentées dans cet article sont le fruit d'une recherche menée dans le cadre du projet international « Strasbourg à la découverte du Nord " (mis en place par Thomas Mohnike et le Département d'études scandinaves de l'Université de Strasbourg, en collaboration avec des chercheurs européens). Le projet a permis, entre autres, la numérisation et la valorisation du fonds scandinave de la Bibliothèque nationale et universitaire de Strasbourg.

2 - Voir à ce sujet Henri Dubled, Histoire de la Bibliothèque nationale et universitaire de Strasbourg, Strasbourg, 1973

3- Grammaticus, Saxo et Pedersen, Christiern, Danorum regum heroumque historice : stilo eleganti a Saxone Grammatico natione Sialandico necnon Roskildensis ecclesice preposito, abhinc supra trecentos annos conscriptee et nunc primum literaria serie illustratce tertissimeque impresse, Paris, 1514

4- Grimm, Jacob et Wilhelm, Lieder der alten Edda. Aus der Handschrift herausgegeben und erklärt durch die Brüder Grimm, Berlin, 1815

5 - Heusler, Andreas, Codex Regius of the Elder Edda, Ms. No $23654^{\circ}$ in the old royal collection in the Royal Library of Copenhagen, in Corpus codicum islandicorum medii aevi, 10, Copenhagen, 1937

Voir aussi Hermannsson, Halldór, Icelandic illuminated manuscripts of the middle ages, in Corpus codicum islandicorum medii aevi, 7, Copenhagen, 1935

6 - Franckenstein, Christian-Gottfried, Histoire des intrigues galantes de la reine Christine de Suède et de sa Cour, pendant son Séjour à Rome, Amsterdam, 1797

7 - Ólafsson, Eggert, Voyage en Islande... Traduit du danois par Gauthier-de-Lapeyronie, traducteur des Voyages de Pallas. 5 tomes, Paris, 1802 